\title{
AESTHETICS: FROM NATURE TO ENGINEERING AND BACK
}

\author{
M. Tanga, F. Ghelli
}

Siena Academy of Sciences

Aesthetics rises in XVIII Century involving the inner and the senses of the subject. The bipolar couple beautiful/ugly is no more enough to define new emerging aesthetic experiences of agreeable, charming, interesting, sublime...

Traditional models of Perfection (based on metron, nomos, logos, eurhythmy, symmetry, once centrepieces of the metaphysics of beauty) are soon overruled: irrational, disgusting, ugliness, darkness, mystery, shadow, troubled, dreadful, perturbing gain wide and independent spaces. Time, body, proximity senses, geographic, naturalistic and technological discoveries and inventions are among the factors of this turning point that go with the development of aesthetics. Rising and growing modern science, new technology and engineering determine a deep crisis in the aesthetic vision of nature, art, world, but also offer the references to build a new cultural and existential frame. In particular, actual engineering contributes to quality of life and offer explanations and demonstrations about how and because a thing or an action can satisfy our aesthetic sense, meant in a wide acceptation. As example we use the geometry of road and its curves and we compare this with structures we find in Nature. We use curves whose curvature changes along their development. They are very suitable to show how science and technology have dispelled the classic idea of perfection (based on quite, simple, exactly defined shapes as circle or square) and have contributed to build a new kind of aesthetic taste that understands and appreciates less defined and more problematic ones, coming from different laws.

Keywords. Aesthetics - Nature - Time - Science - Art - Engineering

\section{ESTETICA E SCIENZA: DEBITORI RECIPROCI}

\author{
"Aeasthetica (artium liberalium Theoria, \\ gnoseologia inferiore, ars pulchre cogitandi, ars analogi rationis) \\ sensitivae cognitionis est scientia." (Baumgarten, 1750)
}

Il bello esiste nella nostra cultura fin dalle sue origini (il concetto di tò kalòn, nell'antica Grecia, era carico di profondi significati ed era associato a quelli di vero e di giusto, ma l'estetica (una disciplina con un suo corpus epistemico autonomo) nasce nella modernità inoltrata, nel XVIII secolo: noi consideriamo il suo atto di nascita l'“Aesthetica" di Baumgarten (1750), ma il termine è un po' più vecchio: lo stesso autore lo usa già nella sua tesi di laurea. In altri autori sono presenti molti concetti connessi con l'estetica moderna. Addison, per esempio, in alcuni articoli per la rivista "The Spectator", nel 1712, parla di "piacere dell'immaginazione".

Con l'estetica l'attenzione non è sul bello in sé, ma sull'esperienza che provoca nel soggetto che con esso ha a che fare. Il soggetto, il suo pensiero, il suo centro di soggettività, guadagnano il primo piano nella concezione della conoscenza e della sua costruzione. Ma il soggetto non è solo pensiero, egli "ha" un corpo, anche...

La cultura occidentale ha sempre o quasi sempre diviso il corpo dalla mente, ha sempre identificato questi due aspetti come due cose reciprocamente estranee, e l'uomo è stato considerato un'entità composta, la cui natura è doppia.

Non solo corpo e mente sono divisi, ma sono anche disposti gerarchicamente, con la mente in posizione preminente.

Quelli delle concezioni materialistiche possono complessivamente essere considerati tentativi che non hanno ottenuto successi duraturi o convincenti nell'invertire questa gerarchia. Sono stati costruiti equilibri instabili che di fatto non hanno cancellato il dualismo mente-corpo.

Sebbene tale posizione diviene talvolta un monismo eliminativo nei riguardi della controparte del corpo (la mente), comunque queste dovevano essere confrontate con gli aspetti più profondi della nostra cultura. E la nostra cultura non può rinunciare alla mente che, se viene eliminata, presto trova il modo di riguadagnare le posizioni perdute.

L'ineliminabile esperienza del sé, l'autocoscienza, la soggettività acquistano importanza proprio nel XVII-XVIII secolo, quando la rivoluzione scientifica colloca la necessità di oggettivare la realtà in primo piano.

La soggettività rimane nel regno dell'anima o della mente e diviene sempre più forte, opponendosi al mondo esterno oggettivo.

Descartes dà un contributo decisivo alla svolta della cultura occidentale in direzione dell'introspezione e del sog-

Correspondence to:

Mario Tanga

Via Giovi, 202/B - 52100 - Arezzo - Italy

Tel.: +39 0575362603 - Cell.: +39 333.133.0333

e-mail:m.tanga@tin.it 
gettivismo. Sulla stessa linea troviamo anche il metodo e la teoria della logica di Port Royal. Descartes di rendere la "res extensa" e la "res cogitans" due cose distinte ma confrontabili. Tuttavia la loro simmetria non è durevole, perché il corpo è pensato e non pensante, è passivo e oggettivo, è misurabile e non "misurante". Esso è irrimediabilmente lontano dalla mente. Al momento non c'erano le condizioni perché divenisse pienamente soggetto.

L'uomo e cosciente di pensare ma anche di percepire (nonostante i dubbi di cui Descartes investe quest'ultima attività). In ogni caso, insieme con il pensiero individuale, i sensi guadagnano sempre più importanza in molti contesti. Soprattutto a partire dal XVII secolo, notiamo un uso sistematico del metodo osservativo e l'affermarsi del sensismo.

Il primo che ha dato un riconoscimento a questo modo di acquisire conoscenza è stato Aristotele (anche se ne troviamo traccia già in Protagora), e, attraverso l'aristotelismo rinascimentale, giunge a confluire nella moderna teoria che ne fa un vero e proprio metodo scientifico: quella di Locke, Hume, Berkeley, Condillac, James.

Fondamentalmente il sensismo è basato sul concetto che "nulla esiste nella mente che prima non sia stato nei sensi" (Locke nel 1690 scrive "Nihil est in intellectu, quod non prius fuerit in sensu").

Non a caso Leibniz poco dopo puntualizzerà: «excipe: nisi intellectus ipse" (Leibniz, 1765), come dire: il principio primo di conoscenza, astratto dal mondo e autoreferenziale, non può essere tagliato fuori o messo nell'angolo, il dominio dei sensi non copre tutto il conoscibile. Il sensismo non coincide affatto con il materialismo: il corpo è materiale e accede al mondo in quanto materiale, ma questo non esclude un'ulteriorità dove si svolgono i giochi della conoscenza da una parte e, un secolo più tardi, quelli del giudizio (estetico).

Come dire che per adesso il sentire "esterno" (tramite i recettori fisiologici) è costitutivo del sentire "interno" (gradimento, piacere, gratificazione, compiacimento, attrazione, emozioni e sentimenti in genere).

Corollario di questo è stato lo sviluppo di studi e ricerche focalizzati sugli aspetti concreti, di uso, di interazione materiale con oggetti, contesti, processi...

Per adesso il corpo solo è punto di passaggio, è rilevatore di dati nel mondo, ma la sua detenzione di questi dati è transitoria. Consegnati alla mente, è qui che saranno usati per generare contenuti cognitivi o giudizi estetici. Ma i sensi, con l'esercizio del rapporto estetico con il mondo, non possono essere a lungo costretti entro lo stretto limite di strumento. Sono stati tirati in ballo ed è questione di tempo perché sviluppino le conseguenze della loro implicazione.

Fondare l'estetica ha messo in moto più di quanto era nell'intenzione dei suoi iniziatori, pertanto possiamo dire che l'estetica moderna (o, tout-court, l'estetica) nasce con un doppio seme, di straordinari sviluppi culturali e di crisi (implosione, frammentazione, dissolvimento dei significati originari) che culminerà nel $\mathrm{XX}$ secolo.

Ma nella seconda metà del Novecento viene anche scoperto tutto un sistema di "effetti crociati" tra la funzione fisiologica, il vissuto corporeo e quello interiore del pensiero e dei sentimenti.

Si apre un orizzonte di ampiezza straordinaria, con implicazioni spesso inattese e sorprendenti.

Tornando al XVIII secolo, fin da allora il focus si sposta dalla bellezza "oggettiva", "esterna", all'interiorità del soggetto. Ed è qui che già da Addison, Burke, Hume, etc., l'estetica incontra le prime difficoltà, nei limiti del soggettivismo, avvisaglia della crisi che deve svilupparsi.

La coppia bipolare "bello/brutto" non riesce a racchiudere la gamma di sensazioni di cui il soggetto ha coscienza. Tale coppia non basta più per definire le nuove esperienze estetiche di piacere, gradevolezza, fascino, interesse, carattere pittoresco, sublime...

L'atto del guardare (dal XVII secolo) acquista consapevolezza e trova il suo scopo in se stesso. Il soggetto che guarda trova piacere e soddisfazione nella sua attività di scoperta. Va a caccia di immagini, visioni che devono essere sempre più belle, sorprendenti, spettacolari, difficili da trovare, persino proibite. Tale soggetto diviene a poco a poco un voyeur, un curioso, un invadente, un inopportuno, un "affamato" di immagini.

Guarda l'artista (la realtà) e guarda lo spettatore dell'opera. L'occhio di entrambi è testimone della medesima scena. Paesaggi, scene di genere, situazioni intime, scabrose, soggetti inusuali, tutto può soddisfare questo desiderio, e quando qualcosa viene scoperto il voyeur ha bisogno di qualcos'altro di più spettacolare, più sorprendente. È la stessa molla che talvolta spinge alla ricerca delle stranezze della natura, di esemplari o fenomeni bizzarri da inventariare, annotare, illustrare, classificare.

Tale tendenza porterà, nel tempo, all'attuale inflazione di immagini (anche grazie alla possibilità tecnica della loro (ri)produzione, cfr. Benjamin, 1966), alla ricerca (anche da parte di cinema e TV) dell'estremo, dell'iperbolico, dello shock, arte e media spesso propongono il "kitch".

Valore, significato e funzione dell'immagine cambiano: l'immagine non è più imitazione che offre un doppio di livello inferiore, una deriva peggiorativa (cfr. Platone), ma comunque capace di filtrare e abbellire ciò che rappresenta (cfr. Aristotele). Ora essa è la creazione di un nuovo e differente mondo, capace di offrire hybris non del vero, ma del verosimile, di far dimenticare il mondo reale, confondendolo con quello fittizio...

Fondare l'approccio estetico con il mondo coincide con la scoperta che possiamo fare più che conoscere il mondo, più che darci una legge morale, possiamo giudicare, scegliere, decidere cosa siamo e come, avere il nostro autonomo spazio di discrezionalità.

Il giudizio si accorda al proprio gusto e orientamento, alla ricerca del piacere, della soddisfazione, e l'uomo è l'unico soggetto di queste decisioni, a conferma del suo potere individuale. Scivolare verso un individualismo egocentrico, nell'Estetismo (XIX-XX secolo) del Dandy, nel solipsismo, perdere il senso di appartenenza alla comunità e l'identità culturale e sociale, questi sono i rischi e i pericoli spesso attuatisi nella nostra società, quando l'originaria libertà e soggettività è stata esasperata e ha degenerato.

La perfezione, cifra della bellezza, una volta modello e riferimento, perno della tradizionale metafisica della bellezza, nella Modernità (e in particolare nel secolo scorso) si è capovolta, divenendo persino negativa, l'opposto della vita, della libertà e del dinamismo.

Logos, metron, nomos, euritmia, simmetria, una volta principali riferimenti della teoria della bellezza (cfr. Policleto, Vitruvio...), sono perduti. Già con Beethoven e Mozart vengono usate le dissonanze. Nella tarda letteratura romantica la malattia, l'anomalia, la mostruosità, 
il vizio, la degenerazione, la turpitudine sono esaltate. A partire dal Manierismo, e dopo con Caravaggio, Goya... ma principalmente con il romanticismo, l'irrazionale, il disgustoso, il brutto, il tenebroso, il mistero, l'ombra, il terrificante, il perturbante conquistano spazi ampi e indipendenti, al di là dell'opposizione dialettica alla bellezza che tutto ciò ha in Rosenkranz ("Aesthetik des Hässlichen", 1853).

Il tempo come dimensione si stabilisce ovunque: tutto è soggetto al cambiamento, dai corpi celesti dell'astronomia galileiana alla teoria geologica delle catastrofi, all'entropia, al darwinismo. Ma questo è un tempo diverso da quello hegeliano, che mirava a una conclusione perfetta, capace di ripristinare l'armonia, l'unità, di condurre il mondo e l'uomo a un livello gerarchicamente superiore.

La storia geologica mostra che esiste un tempo oscuro e profondo, un lungo passato senza l'uomo. Questo è terrificante.

Il principale riferimento parmenideo, la distinzione tra ciò che è (l'ente, l'essere) e ciò che non è (il non-essere), garanzia di verità, ha prevalso nella cultura occidentale. L'eracliteo "tutto scorre" (così come l'unità degli opposti) rimane sullo sfondo, ma adesso la pervasiva dimensione del tempo ritorna, e per di più caricata dell'incertezza del caso.

Determinare entità e isolate, la loro identità, localizzare il loro nucleo, segnare i loro confini, è stata la tendenza dominante della scienza e della filosofia occidentale. Sulle vecchie certezze adesso prevale un differente quadro: il flusso, il processo, la mutevolezza, la variabilità, la metamorfosi... emergono in primo piano negli studi naturali e umanistici.

Molte illustrazioni (a partire dal XVII secolo) ricercano l'"attimo fuggente", colgono un" "istantanea di transizioni più o meno rapide, movimenti, iniziando, tra l'altro, a fare attenzione alle trasformazioni patognomiche del volto e del corpo, all'espressività gestuale e non soltanto alle caratteristiche fisiognomiche, strutturali.

Il corpo, inizialmente condizione di percezione, più tardi (XX secolo) diviene un soggetto che non può essere subordinato o ridotto alla mente o all'anima (Irigaray, Derrida...). I sensi di prossimità (cfr. la Madalaine di Proust in Alla ricerca del tempo perduto) acquistano importanza: il gusto (termine che designa sia il senso che ci fa apprezzare il cibo sia la facoltà di giudicare e scegliere!...) e l'olfatto (dal XVII secolo l'arte del profumo è molto apprezzata, e sia il fascino che il disgusto dell'odore acquistano interesse.

La nudità non richiede più giustificazioni mitologiche o religiose. L'eros e la carne sono in primo piano ( "L'anima nel Barocco intrattiene con il corpo un rapporto complesso: sempre inseparabile dal corpo, trova in quest'ultimo un'animalità che la stordisce, che la impastoia nei ripiegamenti della materia, ma pure un'umanità organica o cerebrale che le permette di innalzarsi, e la farà salire su tutt'altre pieghe.", Deleuze, 2004).

Il dinamismo corporeo diviene fonte di piacere e occasione per produrre/esibire prestazioni.

La natura è un campo di indagine, una sorgente di meraviglia (la ricerca e l'ossessiva attenzione per i vari $m i$ rabilia è sintomatica) da collezionare e studiare. Le cose scientificamente studiate sono anche giudicate esteticamente e viceversa, con reciproca influenza tra le due attività. Ma, per adesso, senza armoniosa integrazione. Eccezioni e stranezze sono preferite alle cose usuali, regolari.
L'esplorazione di continenti esotici e lontani amplia gli orizzonti della Modernità. La natura mostra anche il suo lato oscuro e selvaggio, il pericolo, il pauroso, le terribili profondità, offre brividi (hybris) e richiede coraggio.

La dimensione prometeica dell'uomo che affronta tale natura può essere trovata sia nella sua anima sia nel suo corpo (cfr. Burke, 1757).

Nel XX secolo il giudizio estetico si estende a campi fino ad allora inesplorati, oltre il visuale (delle arti e della natura) e l'uditivo (della musica), fino ad essere riferito alla vita (anche nell'accezione politica del termine), alla forma (della comunicazione e quindi ai media), alla conoscenza (in chiave criticistica e scettica), al sentire (fin nelle sue implicazioni fisiologiche). La scienza e la metodologia hanno gran peso in molti di questi campi, soprattutto nello studio della forma (scienza, tecnologia e ingegneria dell'informazione), della conoscenza (metodologia), del sentire (lo studio della fisiologia e della neurofisiologia, i cui dati sono sempre di più interfacciati e correlati con quelli mentali, cognitivi, emotivi, culturali). In particolare lo studio della sensorialità, che ha rivelato un numero di sensi (se di sensi si può ancora parlare) ben superiore ai cinque aristotelici. La sensorialità legata all'esperienza corporea di movimento, di auto percezione, di interazione con il mondo, può essere inscritta nei paradigmi di apprezzamento (estetico) al pari di ciò che vediamo o udiamo. Sensazioni (o insiemi di sensazioni) come la propriocezione, la cinestesi, la vezione possono offrirci un vissuto gradevole o sgradevole, intenso o scialbo, stimolante o demotivante.

Ciononostante la caratteristica distintiva dell'estetica era originariamente definita (cfr. Kant) come altro dall'utile (al contrario di filosofie orientali) e dalla conoscenza. La scienza e la tecnologia che si affermano costruiscono una nuova visione del mondo, ci danno un'accezione diversa del senso estetico.

Quando Galileo mostra che persino le stelle muoiono, che i corpi celesti non sono perfetti e sono omogenei al mondo sublunare, il vecchio mito della perfezione celeste è infranto. Quando Keplero dimostra che il moto dei pianeti non è uniforme, che le orbite degli stessi non sono circolari, le vecchie regolarità geometriche sono dissolte.

I dispositivi ottici (microscopio e telescopio) spostano i limiti dimensionali e di distanza (micro e macro) oltre il naturalmente visibile, l'uomo non è più copula mundi, di un mondo i cui estremi erano comunque commensurabilmente vicini. Il senso della misura e dell'equilibrio è perduto.

Il tempo rende tutto transitorio, dalle stelle ai viventi, alla materia, nella quale vuoto e discontinuità divengono prevalenti (teoria atomica), inoltre, con la fisica quantistica, "Natura facit saltus"!

La teleologia e i principi ordinatori in senso tradizionale sono persi.

Solo nella seconda metà del XX secolo si ha un vero punto di svolta.

Per esempio, quando la scienza ci mostra la similarità fra le strutture di una colonia batterica, di una galassia, una mappa di router di internet e una pittura di Pollock, tutte riconducibili al modello di un frattale, noi non possiamo ignorarlo e ciò ha inevitabilmente influenza sui nostri gusti, sul nostro modo di giudicare la natura, l'arte, il mondo.

La teoria della complessità e i modelli olistici ci mo- 
strano un altro genere di bellezza.

La psicoanalisi rompe la originaria unitarietà del soggetto. Cento anni fa o poco più Freud (1998) mostrava come le pulsioni di vita (libido/Eros, sopravvivenza, propagazione, fame, sete, sesso) e le pulsioni di morte (Tanathos) influenzano le nostre preferenza e guidano il nostro comportamento, non di rado in conflitto una contro l'altra, come l'A. mostra nel suo lavoro "Oltre il principio del piacere". L'inconscio ha il suo ruolo insospettato, ciascuno di noi è teatro di conflitti tra forze in parte sconosciute...

La medicina, d'altro canto offre una ben articolata visione di malattie, patologie e difetti, ma mostra anche come la salute non sia assoluta assenza di essi, non sia perfezione.

La condizione postumana rende il mondo naturale lontano e i significati tradizionali incerti. Le realtà vicarie, fittizie, virtuali, ci rendono "sdoppiati" e capovolgono il mondo: il reale e la finzione si scambiano, i sensi divengono "nudi sensi" se non integrati con dispositivi tecnologici (il cyborg non è più solo fantascienza) che rimpiazzano facoltà mancanti, amplificano o modificano quelle naturali. Per esempio la fotografia nel XIX secolo e il cinema o le tecnologie di imaging animata nel XX. Occhio e obiettivo divengono un sistema unico. Dacché usiamo obiettivi fotografici e cinematografici, non guardiamo più il mondo nello stesso modo, anche se non li stiamo usando concretamente. Siamo così abituati a guardare in uno schermo (apparecchi di ripresa sono ovunque) che persino il mondo reale può sembrare un film...

Le tecnologie di imaging rendono visibile l'invisibile, e possibile l'impossibile, sciolgono la cifra di realtà indefinite, ci danno la certezza strumentale: è sempre più difficile rinunciare a questa amplificazione dei nostri poteri.

L'occhio biologico "vedeva il mondo", ma adesso l" occhio nudo" è inevitabilmente superato dai dispositivi ottici. Riassumendo, questa possibilità di andare lontano, oltre la natura è attuata in ogni senso dal cinema, dalla TV e dalle tecnologie di sintesi grafica digitale: In senso geometrico: con la rappresentazione di parti interne, lontane dalla superficie, misurando la loro dimensione, la loro profondità...

In senso dimensionale: amplificando l'infinitamente piccolo con il microscopio, e non solo quello ottico.

In senso psichico: oggettivando gli aspetti invisibili o elusivi come le emozioni e la cognizione.

In senso cronologico: una ripresa video è una memoria artificiale che può essere differita nel tempo.

In senso spaziale: posso osservare un evento molto lontano da me nello istante in cui succede.

L'integrazione tra il sistema sensoriale (che include sia il recettore periferico che la componente cerebrale) e il dispositivo tecnologico è implementato da modificazioni neurali e cognitive, così che nell'esperienza è molto difficile distinguere tra i due.

L'interazione sensomotoria con dispositivi artificiali è sempre più estesa e articolata, espandendo il nostro dominio ma confondendoci, dandoci un senso di estraneità.

Come esito di ciò, nel XX secolo i principali assi dell'esperienza estetica sono dissolti:

Poiesis: l'uomo non si sente più autore (creatore) di nulla che abbia un'aura (cfr. Benjamin, 1966). La frammentazione della produzione (post)industriale, così come il linguaggio cinematografico, richiedono di es- sere diretti dall'esterno, con un lavoro di montaggio affinché abbiano un senso.

Aisthesis: i sensi hanno bisogno degli ausili tecnologici, che però ci fanno perdere la diretta connessione al mondo.

Katarsis: la trascendenza dell'identità, della relazione con se stessi, portano a confusione esistenziale, al disorientamento, a obiettivi indecifrabili.

Ricordando la famosa frase di Rimbaud"Io è un altro", il nostro pensiero va a molte terribili esperienze, dalla schizofrenia all'anoressia (non a caso tipica della società occidentale contemporanea) dove il corpo, la sua consistenza materiale è l'estraneo, il nemico.

L'anoressia è tutt'altro che un edonistico desiderio di essere agili, snelli e desiderabili. È ben di più che un semplice disturbo del comportamento alimentare: è un problema globale, esistenziale, profondo, ha capovolto l'approccio estetico al corpo, l'aspirazione erotica di essere desiderati e desideranti. Thanatos ha vinto su Eros... Distruggere, dissolvere, annientare il corpo è il perverso "contropiacere", principale scopo dell'anoressia. Solo dopo la metà del XX secolo il corpo guadagna un ruolo primario: superando i pesanti limiti sia dell'idealismo che del determinismo materialista, il corpo è un essenziale aspetto della soggettività e del soggetto. Nei processi cognitivi, così come in quelli emozionali, relazionali o estetici, la modalità corporea non può essere esclusa: non possiamo vivere né capire simili esperienze a prescindere dal corpo.

Oggi parliamo di neuro estetica, sensologia, estesiologia comprendendo l'accezione scientifico-medica di tali termini), il corpo ha pienamente acquisito un ruolo primario. Il S.N., l'informazione biologica, l'interazione fisica con l'ambiente (inteso come umwelt) sono i termini per parlare di una reciproca inclusione (introdotta dalla fenomenologia) tra il soggetto e il mondo, tra la mente e il corpo, secondo le nuove teorie dell'Esternalismo, la Conoscenza Incarnata e Radicata (EEC, dall'inglese Embodied Embedded Cognition), il Comportamento Interattivo Immediato (IIB, Immediate Interactive Behaviour) e così via, che dischiudono nuovi orizzonti culturali ed esistenziali.

Il nostro cervello è il nostro corpo (esattamente una sua parte), ma non solo. Il cervello è una parte molto particolare, grazie alla quale percepiamo, conosciamo e gestiamo coscientemente il nostro corpo, viviamo il nostro corpo, siamo il nostro corpo. vivere il corpo attraverso la mente-cervello è l'unico modo per viverlo... Non c'è dualismo tra il cervello e il corpo (somatico), tra controllore e controllato. Al più essi sono differenti aspetti della stessa cosa. Possiamo considerarli come indistinguibilmente integrati uno con l'altro.

I processi evolutivi, adattivi, di sviluppo, di apprendimento, e persino culturali non possono essere concepiti senza il corpo, le sue percezioni, le sue interazioni fisiche, la sua collocazione nel mondo (umwelt). Il nostro corpo sarebbe una semplice "cosa" se non fosse pensante/pensato (pensiamo anche con il corpo!...), se non gestisse il/fosse gestito dal cervello, se non fosse immerso nella cultura.

Il corpo è sempre impegnato in uno scambio con il mondo che è insieme fisico e informazionale/semiotico. La retro propagazione dell'errore (cfr. il connessionismo) ha una natura corporea ed è condizione essenziale per rendere il cervello capace di interagire con il mondo. La micro- e macro-architettura del cervello acquista specificità per ottimizzare le interazioni future, 
così come è atteso che si verifichino.

Ciò è possibile grazie all'inclusione del mondo (esterno) nella funzionalità cerebrale.

Dobbiamo superare la teoria puramente proiettiva dell'homunculus. Anche se esiste un importante sistema di connessioni (cervello-corpo e corpo-cervello), ciò non è né l'unico né il principale modo di unire "centro" e "periferia".

Possiamo notare che il corpo insieme con i dispositivi esterni è un sistema nuovo e differente, che emerge dalla reciproca interconnessione. Tale connessione è molto stretta, non è una semplice giustapposizione, e può essere definita "accoppiamento strutturale" $\mathrm{o}$ in modo simile.

Il nuovo sistema che in virtù di essa emerge non è "autocentrato", talvolta nemmeno "neurocentrato". I processi di apprendimento trasformano l'interazione occasionale e casuale in una inclusione del mondo esterno nel corpo.

Persino processi tipicamente cerebrali come il rievocare sono basati solo su risorse interne. Il cervello "fa conto" nei riferimenti offerti da opportuni oggetti/eventi esterni. I dati del mondo esterno sono processati insieme e nello stesso modo dei dati del corpo. È molto difficile distinguere l'oggetto esterno come tale: lo percepiamo in continuità e omogeneità con il nostro corpo. l'apprendimento è la confluenza dei dati interni ed esterni in un unico sistema computazionale. Tramite l'apprendimento acquistiamo familiarità con nuove situazioni, correlando opportunamente l'input con l'output (cfr. P.M. Churchland, 1992).

Siamo inestricabilmente connessi con il mondo; i trasduttori sensoriali e l'apparato esecutivo possono essere considerati solo un differente tipo di sinapsi, attraversate da un flusso di informazioni provenienti da e dirette al mondo: $\mathrm{i}$ "Memi" non abitano nel chiuso della nostra scatola cranica, essi fluiscono, tra la mente/cervello e il corpo, tra il corpo e il mondo in ambo i sensi.

Oggi non possiamo più separare concezioni scientifiche ed esperienza estetica. La scienza ci dà le basi per una rivoluzione tutt'altro che conclusa. Alcune frasi possono riassumere questa svolta epocale:

Il sistema diviene prevalente sul singolo elemento. L'intero diviene prevalente sulla parte.

La connessione diviene prevalente sul nodo isolato. L'oikos diviene prevalente sull'ontos.

L'emergere diviene prevalente sulla causazione deterministica.

La codeterminazione diviene prevalente sul determinismo lineare.

La complessità diviene prevalente sull'ordine rigido e sul caos stocastico.

L'autorganizzazione diviene prevalente sulla catena causa-effetto.

L'omeorresi diviene prevalente sull'omeostasi.

La commistione sfumata diviene prevalente sul rigido confine.

L'estetica attuale deve procedere in questa direzione: $\mathrm{i}$ vecchi dualismi sono superati. Coppie come bello/brutto, soggetto/oggetto, esterno/interno divengono sempre più ricche e articolate fino a perdere i significati originari, grazie a ulteriori e differenti concetti che configurano un nuovo e complesso quadro teoretico, euristico ed estetico.

Nel XX secolo la dimensione corporea dell'estetica raggiunge la sua completa crisi, ma anche fa intravedere nuove prospettive future.

Come Sean B. Carrol suggerisce nel titolo del suo libro sulla biologia Evo-Devo "Infinite forme bellissime" noi possiamo insieme conoscere (scientificamente) e godere (esteticamente) nuovi mondi.

\section{L'INGEGNERIA TRA COMPRENSIONE DELLA NATURA E PROGETTAZIONE}

Tale nuova direzione è indicata da molti esempi. Uno di essi può essere considerato la nascita della ergonomia contemporanea a Oxford, nel 1949, che inaugura un filone di ricerca su base medico-ingegneristica finalizzato a orientare la progettazione (compreso il design) al soddisfacimento di esigenze psico-fisico-relazionali. L'ingegneria estende il suo campo, non di rado interferendo in quelli che tradizionalmente erano i domini di azione dell'architettura e delle "belle arti" in genere.

La contemplazione e l'“aisthesis" non sono più l'unico e nemmeno il principale modo di rapportarsi con l'oggetto.

La staticità lascia il posto al dinamismo, il prodotto al processo, la contemplazione all'azione, la struttura alla funzione, e via dicendo.

In particolare l'aspetto processuale e la sua valorizzazione ha radici che potremmo far risalire addirittura al tardo Medioevo: si pensi alla tecnica di costruzione della cupola del Duomo di Firenze senza ricorrere a centine. Oltre che progettare il prodotto l'ingegneria progetta anche e sempre di più il processo, processo che ha un contenuto tecnologico e teoretico sempre più elevato. Nella modernità avanzata, e soprattutto dal XIX secolo, la conoscenza diviene sempre più fonte di piacere e gratificazione. Un'altra barriera che dovrebbe isolare l'estetica nella sua torre d'avorio (separandola in questo caso dalla conoscenza) è infranta.

In tutto questo trova spazio l'ingegneria che costruisce e offre gli strumenti di formalizzazione, controllo e gestione di produzione e uso degli oggetti.

Dall'ingegneria vengono le spiegazioni e le indicazioni di come e perché un oggetto è o può essere piacevole, gradevole, elegante...

Ingegnerizzare i rapporti con la realtà non significa avere la garanzia dell'infallibilità, anzi significa proprio lasciare all' errore spazio e assegnargli una funzione informativa, farne un elemento di confronto.

Il vissuto estetico oggi non può prescindere dalla semplicità di uso dell'oggetto, dalla qualità (anche fisica, corporea) dell'esperienza di interazione, dalle implicazioni funzionali e di utilità che tale interazione mette in gioco. Semplicità come eleganza, come pulizia, come purezza, come assenza di disturbi, come economia di tempo e risorse attentive per le caratteristiche intrinseche dell'oggetto e quindi come possibilità di concentrarsi su aspetti esterni e sovraordinati, quindi come libertà e apertura del pensiero.

Un esempio paradigmatico è il mouse prodotto dalla Apple ("Magic Mouse") nel 2009, che presenta una superficie compatta, intera, senza alcun elemento di azionamento meccanico, come erano i tradizionali tasti (sx e dx), e la rotellina di scorrimento. Il lembo di superficie superiore è di fatto un "touch-pad" interattivo, con ulteriori funzioni. Il tipo di contatto (ampiezza, direzione, velocità...) calibra e determina le azioni "sfoglia pagina", "zoom" di pagina o di schermo, "scorri pagina", e in genere tutte le funzioni tradizionalmente affidate ai tasti $\mathrm{dx}$ e sx. Le vere novità sono che tutta la 
superficie è recettiva in tal senso (e quindi non ci obbliga a azionare in modo mirato questo o quel comando), inoltre i gesti di "touch" sono molto più intuitivi e accettano un margine di approssimazione maggiore, senza perdere efficacia e precisione, infine la gamma di comandi è molto più articolata e consente di evitare in molti casi lo spostamento fisico del mouse sul pad.

Questo è ottenuto grazie a una tecnologia sempre più evoluta e che è presente in sempre maggior quantità all'interno dell'oggetto, ma per offrire un'interfaccia sempre più conformata sulle esigenze e i gusti del soggetto. E pur vero che l'oggetto diventa sempre più una "scatola nera" e si segna un divario sempre maggiore tra la conoscenza del fruitore (relativa all'uso) e la natura dell'oggetto, sempre più inaccessibile, ma il vissuto è sempre più soddisfacente in termini che possiamo definire insieme "estetici" e "funzionali".

La vera innovazione è che questa nuova estetica si interessa di oggetti che tradizionalmente sono stati non solo ignorati, ma anche ritenuti tutt'altro che degni di considerazioni di questo tipo. Cosa c'è di "bello" o esteticamente apprezzabile in un mouse? In una curva di un tracciato stradale?

La prima cosa che notiamo (che l'ingegneria ci fa notare) è la stupefacente (dobbiamo davvero stupirci?) analogia tra strutture naturali (del mondo biologico o minerale) e strutture ingegneristiche, la convergenza tra le soluzioni cui è giunta l'evoluzione biologica o a cui portano i processi entropici da una parte e quelle messe a punto dall'ingegneria dall'altra. Tale convergenza viene notata spesso a posteriori, come dire che l'ingegneria giunge a tali soluzioni, analoghe a quelle naturali, indipendentemente da esse.

Questa può essere se non una prova almeno un indizio a sostegno del fatto che le soluzioni in questione [es. l'impacchettamento esagonale delle schiume, dell'alveare, le reti metalliche di contenimento degli argini, o briglie] rappresentano un optimum a prescindere. In seguito alla constatazione di tali convergenze, oggi l'ingegneria sempre di più cerca zone di intersezione con le scienze naturali. Sembra evidente che questo interloquire tra l'ingegneria (progettuale e prescrittive) e le scienze naturali (descrittive ed esplicative) sia solo all'inizio e molto resti ancora da fare su questa strada.

Nell'attualità la demarcazione tra utilità, modo di fruizione, funzionalità, convenienza da una parte e bellezza dall'altra non è più sostenibile nei termini tradizionali che facevano della mancanza di implicazioni pratiche l'elemento definitorio della bellezza: "un oggetto è bello se prescinde dall'utile".

Per dimostrare quanto ormai bellezza e utilità siano indissolubilmente legate, segnando un decisivo salto di qualità rispetto alla visione dell'estetica del XVIII secolo, possiamo usare innumerevoli esempi, che ci offre l'ingegneria.

Uno di questi esempi significativi di convergenza di utilità e bellezza lo possiamo trovare nello sviluppo plano-altimetrico di una sovrastruttura stradale. L'aspetto altimetrico sarà qui solo accennato per brevità di esposizione.

Il percorso della strada, per molti e differenti motivi, presenta uno sviluppo non rettilineo.

In natura troviamo molti esempi di curvatura che possono offrire significativi termini di confronto.

La matematica, la fisica e l'ingegneria hanno costruito un interessante paradigma concettuale che consente di superare la vecchia dicotomia "naturale-artificiale" non con un semplice sincretismo o con una banale analogia o con un rimando semantico, bensì dimostrando come si possa riconoscere e quindi ricondurre a un modello matematico una struttura fisica o biologica e una antropica.

Il problema della curvatura lo troviamo, è l'esempio comparativo che scegliamo, nell'anatomia dei molluschi, in particolare dei gasteropodi. Probabilmente per ragioni di "compattamento dimensionale" forse per essere meno esposti ai predatori, l'evoluzione ha favorito massicciamente l'avvolgimento elico-spirale delle parti molli e del guscio da esse formato e che le protegge.

Infatti, la torsione viscerale e la conseguente torsione conchiliare dei molluschi gasteropodi, di alcuni cefalopodi e, anche se in modo meno evidente, dei bivalvi segue un andamento particolare che, guarda caso, è "imparentato" con la tracciatura delle nostre strade.

La conchiglia soddisfa esigenze biologico-adattive del mollusco, ma attira anche la nostra meravigliata ammirazione. La conformazione della strada non ha forse lo stesso tipo di attrattiva, ma ugualmente ha dei pregi estetici, come vedremo, che, al di là dell'eleganza della formulazione matematica, offre piaceri percettivi e motori, in accordo con quelle che sono oggi le concezioni dell'estetica.

Cominciando a porre il problema della tracciatura di una strada, il primo elemento che intuitivamente (e rozzamente) possiamo usare è un insieme di segmenti variamente dimensionati e variamente angolati.

Per la schematizzazione di una sovrastruttura stradale, scegliendo di rappresentarla con il suo asse (all'interno di un piano cartesiano georeferenziato), possiamo infatti utilizzare una linea poligonale irregolare che tenga conto, questo sì, della morfologia del territorio indicata dalle curve di livello. Questo tipo di tracciatura richiede solo l'uso del righello. La strada così fatta sarebbe impercorribile!...

Un secondo approccio, tutt'oggi utilizzato nelle strade a basso scorrimento e nei piani di zonizzazione primaria, per ragioni di semplicità ed economia (anche se nulla vieterebbe di ricorrere a modelli più evoluti, come quello che si va ad esporre), che affina quello suesposto, è quello di raccordare gli angoli della poligonale tramite archi di circonferenze tangenti ai segmenti, il cui centro è ovviamente collocato nell'angolo convesso che a due a due, disposti contiguamente, essi formano. Ma anche questa combinazione di segmenti e parti di circonferenza non ottimizza la percorrenza, lasciando un ampio margine di pericolo e di disagio all'utente, soprattutto perché si passa istantaneamente da una curvatura zero a una curvatura determinata e quindi con una derivata dell'accelerazione (contraccolpo) infinita. A basse velocità il fatto è pressoché trascurabile. I problemi iniziano con velocità sostenute. Pertanto occorre andare oltre!... ed è qui che le curve conciliari fanno il loro significativo ingresso, a sostegno di quella visione olistica degli oggetti naturali e artificiali cui si accennava.

Nella progettazione di sovrastrutture stradali (tipologia " $\mathrm{A}$ ", cioè autostrade, superstrade...), l'elevata velocità di percorrenza dei veicoli richiede un modello più evoluto di quello segmento-arco.

In tale modello si sceglie di realizzare il raccordo tra l'elemento geometrico rettifilo (segmento di retta) e lo sviluppo di una curva a raggio fisso (arco di circonferenza) attraverso l'impiego di una curva a raggio varia- 
bile, appartenente alla famiglia delle spirali logaritmiche, di equazione

$$
r \cdot s^{n}=A^{n+1} \text {, dove }
$$

$r$ è il raggio della curva nel punto considerato,

$s$ non è altro che lo sviluppo della curva nel punto considerato, ovvero l'ascissa curvilinea,

$n$ è il coefficiente di forma che rappresenta di quanto varia la curvatura $(1 / r)$ al variare di $s$, ovvero dello sviluppo.

I tre casi limite sono

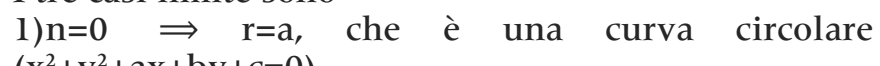
$\left(\mathrm{x}^{2}+\mathrm{y}^{2}+\mathrm{ax}+\mathrm{by}+\mathrm{c}=0\right)$

2) $\mathrm{n}=\infty \Rightarrow \mathrm{r}=\infty$, che è una retta $(\mathrm{y}=\mathrm{mx}+\mathrm{q})$

3) $\mathrm{n}=\mathrm{l} \Longrightarrow \mathrm{r} \cdot \mathrm{s}=\mathrm{A}^{2}$, che è una clotoide, ovvero un caso particolare di spirale logaritmica.

Di questi tre la scelta cade sul terzo perché rappresenta l'entità fisico-geometrica adatta a realizzare il raccordo tra elementi a curvatura diversa, grazie alla variazione punto-punto del suo centro di istantanea rotazione (e quindi anche del raggio) lungo l'intero sviluppo dell'asse stradale. In coerenza a tutto ciò, anche la rotazione della piattaforma stradale viene effettuata all'interno della curva di transizione in modo che la transizione dell'asse stradale corrisponda anche la transizione della piattaforma. La piattaforma, infatti, dotata di ingombro, richiede uno studio sul comportamento ed evoluzione dei cigli (margine sx e dx), e questo anche per evitare fenomeni di ristagno dell'acqua con conseguente acqua-planning (effetto Orn).

Tutto ciò è necessario perché il semplice inserimento di un arco di circonferenza tangente al rettifilo non tiene conto del fatto che su un tracciato stradale ci sono veicoli in movimento a velocità elevata e per questo sottoposti a diverse considerevoli forze fisiche, in particolar modo all'accelerazione centrifuga il cui modulo

è espresso dalla relazione $a_{c}=\frac{v^{2}}{r}\left\lceil\frac{m}{\sec ^{2}}\right\rceil$.

Un rettifilo può essere pensato come una porzione di circonferenza il cui centro è posto all'infinito, per cui, come riscontrabile nella pratica, il veicolo è sottoposto

solo ad accelerazione longitudinale, poiché $\overrightarrow{a_{c}}=0$.

Una circonferenza dal canto suo presenta un raggio definito e quindi un centro identificabile fisicamente, ad es. con coordinate cartesiane assolute o parziali $(\mathrm{x} ; \mathrm{y})$,

il quale fa sì che $\vec{a}_{c} \neq 0$ e, nell'ipotesi che la velocità si mantenga costante, l'accelerazione faccia altrettanto.

Tralasciando l'importanza del contributo dato dalla ovra pendenza della carreggiata stradale e dal fenomeno dell'aderenza trasversale lungo la percorrenza di un tratto di curva, utilizzate per compensare la spinta della forza centrifuga, possiamo esaminare il ruolo del contraccolpo $[c]$.

La grandezza del contraccolpo nell'ipotesi di velocità costante, di essere così definito:

$$
c=\frac{\partial a_{c}}{\partial t}=\frac{\partial\left(\frac{v^{2}}{r}\right)}{\partial\left(\frac{s}{v}\right)}=v^{2} \frac{\partial\left(\frac{1}{r}\right)}{\frac{1}{v} \partial s}=v^{3} \frac{\partial\left(\frac{s}{A^{2}}\right)}{\partial s}=\frac{v^{3}}{A^{2}}
$$

L'unico elemento geometrico che consenta un passaggio graduale da accelerazione nulla ad accelerazione definita, è rappresentato dalla figura geometrica della clotoide in cui, per ogni punto appartenente al suo sviluppo, il centro di rotazione cambia consentendo una variazione graduale e controllata della forza dell'accelerazione centrifuga.

Per cui il contraccolpo, applicato puntualmente negli ipotetici punti di attacco retta-cerchio e cerchio-retta, può essere "spalmato" con una funzione ad andamento lineare lungo lo sviluppo dell'elemento di transizione, rappresentato proprio dalla clotoide, in cui il centro di istantanea rotazione cambia punto per punto, facendo variare gradualmente la accelerazione centrifuga, sia nella fase di ingresso in curva che nella fase di uscita. Come si traduce questo nella fase di progetto? Dettate delle limitazioni sul $c_{\max }$ e sulla $v_{\max }$ di percorrenza della sovrastruttura, ne derivano le limitazioni sul parametro A e quindi, per la $r \cdot s=A^{2}$, sullo sviluppo della clotoide stessa in base alla sua equazione reologica.

La questione sembrerebbe semplice, ma in realtà il vero problema dell'inserimento è costituito dallo scostamento iniziale tra cerchio e rettifilo.

Tale problema può essere risolto:

1) esprimendo l'equazione parametrica della clotoide in funzione dell'angolo al vertice $\tau$, da cui

$\tau=\frac{1}{2} \frac{s^{2}}{A^{2}}=\frac{A^{2}}{2 r^{2}}$;

2) ricavando l'espressione della curva di transizione in coordinate cartesiane.

Si rende necessario definire $d x$ e $d y$ in funzione dell'angolo al vertice $\tau$, risolvendo il sistema algebrico di coordinate del punto generico $\mathrm{P}$ appartenente alla clotoide con uno sviluppo in serie di Taylor ed arrestandolo al primo ordine che corrisponde ad un errore accettabile (ingegneristicamente parlando) dell'ordine del mm;

3) si arriva alla determinazione della grandezza fisica cercata, ovvero lo scostamento indicato con $\Delta \mathrm{r}$.

Questo parametro è stimato attraverso la relazione

$\Delta r \cong \frac{A^{4}}{24 r^{3}}$

arrestando lo sviluppo in serie di Taylor al primo termine.

Tutto questo, che pare un'elucubrazione astratta e poco pertinente, ha invece delle importanti ricadute nell'esperienza di uso della strada, quando la percorriamo guidando un veicolo qualunque.

Oltre al maggior margine di sicurezza che viene garantito sul singolo elemento (ma che tuttavia è già contemplato dall'esame del diagramma delle velocità del tracciato nel suo insieme), si nota che vengono soddisfatti dei parametri che possiamo porre in relazione con il lato "estetico" dell'esperienza di guida.

Si tratta della piacevolezza e della facilità di conduzione del veicolo, della tempistica dell'azionamento dei comandi, oltre che dell'assenza di ambiguità o disorientamento. Quest'ultimo aspetto è legato alla percezione e ai processi cognitivi che consentono di anticipare, programmare, eseguire e supervisionare tali operazioni nel loro insieme.

Il nostro S.N, i nostri recettori e analizzatori, il nostro apparato locomotore hanno delle loro caratteristiche strutturali e funzionali, irriducibili ad aspetti puramente concettuali. Se tali caratteristiche vengono rispettate e sollecitate in modo ottimale (anche tramite un'opportuna configurazione della sede stradale, appunto) questo corrisponde ad una esperienza piacevole 
e gratificante.

Pur se tale aspetto estetico dell'esperienza conserva un quid difficilmente definibile, tuttavia possiamo trovare dei modelli matematici che lo esprimono.

Il primo è percettivo (ottico: visione della curva) e il secondo è motorio (velocità di sterzatura).

Viene definito "criterio di guida ottica", in cui per una corretta percezione della prospettiva stradale la curva di transizione deve possedere uno sviluppo minimo oltre il quale non si provochi disagio ottico, ovvero se la clotoide ha una lunghezza piccola, l'angolo al centro è piccolo e quindi è piccolo anche l'angolo di deviazione. Affinché una clotoide sia otticamente individuabile, l'angolo $\tau$ deve essere $\geq 30^{\circ}$ nel punto di tangenza con la curva circolare. Questo implica che $A \geq(1 / 3)$ r. Dall'altro canto se la clotoide è molto lunga non si riesce a percepire la presenza dell'arco di circonferenza. Vuol dire che si è portati ad arrivare su tale arco con velocità troppo elevata, da cui, limitazione superiore, $\mathrm{A} \leq \mathrm{r}$.

Indicando con $\mathrm{P}$ il passo del veicolo (distanza tra asse anteriore e posteriore), definito con $\theta$ l'angolo di sterzo e con $r$ il raggio della curva, si ha che

$P=r \theta \Rightarrow \theta=\frac{P}{r}$, dove $r=\frac{A^{2}}{s}$,

la variazione di $\theta$ con il tempo porta a definire

$P=r \cdot \theta$

$P=\frac{A^{2}}{s} \cdot \theta$

$\theta=\frac{P \cdot s}{A^{2}} \Rightarrow \frac{\partial \theta}{\partial t}=\frac{\partial}{\partial t}\left(\frac{P \cdot s}{A^{2}}\right)=\frac{P}{A^{2}} \frac{\partial s}{\partial t}=\frac{P}{A^{2}} v$

$\dot{\theta}=\frac{P}{A^{2}} v$

Questo ci dice la velocità angolare con cui muovo lo sterzo è proporzionale alla velocità lineare del veicolo e inversamente proporzionale al quadrato del parametro A della clotoide.

Questo modo di concepire, realizzare e usare un tracciato stradale trova una sua generalizzazione in alcuni casi più complessi.

Oltre che raccordare un rettilineo ad una curva o viceversa (da cui il nome curva "di transizione") si assiste a necessità ulteriori che la realtà ci presenta:

a) Raccordo tra due curve (con centri distinti) nello stesso senso e consecutive, oppure

b) Raccordo tra due curve con verso di sterzata opposto senza lo spazio sufficiente per interporre un rettifilo tra esse.

Nel primo caso si ha una "clotoide di continuità", mentre nel secondo si ha una "clotoide di flesso". Tra le due la più interessante è la seconda perché presenta un cambio di convessità e una schematizzazione geometrica affascinante per la disposizione dei centri di istantanea rotazione.

Questa nomenclatura ci permette di rileggere in chiave ingegneristica le strutture conchiliari. Possiamo prendere come esempi alcuni gusci di conchiglia. Sono particolarmente interessanti alcuni cefalopodi del tardo Cretaceo, il cui avvolgimento piano-spirale presenta molte e bizzarre variazioni di curvatura. Tali gusci, denominati scaficono, amiticono, baculicono ed appartenenti a diversi generi, hanno andamenti che "fanno spreco" di tali curve di raccordo.

Se poi si osserva la sezione di una valva dorsale di un Lamellibranco, genere Pectinidae, con guscio pianoconvesso, si nota che la valva dorsale, approssimativamente piana, in realtà è concava nei pressi dell'umbone, in prossimità cioè della cerniera, mente è leggermente convessa nella parte distale. È presente quindi un flesso a tangente orizzontale, nella parte intermedia della valva.

Altro esempio di clotoide è dato dalla curvatura in proiezione sagittale dei condili femorali. Il raggio di tale curvatura varia e varia il centro di rotazione, in relazione alla fisiologia dell'articolazione, in particolar modo della tensione dei legamenti e della combinazione mobilità-stabilità del ginocchio stesso.

Se un approccio matematico basato sull'esame degli "stress pattern" sembrerebbe di maggior pertinenza, si è preferito l'esame strutturale perché il paragone con la progettazione ingegneristica (in questo caso stradale) consente pienamente di analizzare, per es., lo sviluppo elico-spirale di una conchiglia con altri strumenti che ci fanno meglio comprendere gli atti di moto assiali (rotazione, traslazione, espansione) infinitesimi che la generano e ce la fanno meglio rappresentare in 3D.

Ciò è possibile perché da un punto di vista matematico una figura non perfetta, la clotoide, può essere scelta come elemento di raccordo per lo sviluppo di due elementi perfetti (il cerchio e la retta), in quanto può essere intesa come un insieme infinito di cerchi con l'errore di approssimazione che tende a zero.

La riflessione ingegneristica, che l'esperienza e l'utilizzo ci porta a verificare, non aspetta più di constatare coincidenze a posteriori, ma si dà come criterio euristico primario l'esame della natura, in questo viaggio andata e ritorno che unisce il naturale e l'artificiale.

La stasi del mondo classico è definitivamente infranta, ma la scienza e, con essa la tecnologia, hanno nello stesso tempo lavorato per costruire un altro tipo di bellezza. Con i dovuti tempi si è capito che l'ellisse kepleriana, per es., non era solo una negazione delle sfere e dei cerchi, ma anche l'affermazione di forme nuove, diverse, animate da dinamismi fino ad allora sconosciuti, aperte verso orizzonti che in un primo tempo avrebbero spaventato. Oggi consideriamo "bello" un frattale, o la sensazione tattile e motoria offerte da un oggetto tecnologico ben realizzato, o un procedimento cognitivo e/o costruttivo: le frontiere dell'estetica hanno traslato quantitativamente e qualitativamente in modo e misura tali da essere impensabili nel XVIII secolo.

\section{Bibliografia}

1. AA.VV., Catalogo della mostra L'anima e il volto(Milano dal 30 Ottobre 1998 al 14 Marzo 1999, con opere da Leonardo a Bacon), Milano, Electa, 1998

2. AA.VV., Mostri \& Co., Roma, Newton \& Compton, 2003

3. Abruzzese A., La grande scimmia, Roma, Napoleone, 1997

4. Agostinacchio M., Ciampa D. \& Olita S., La progettazione delle strade, Roma, EPC Libri, 2002

5. Angelucci D. (a cura di), Estetica e cinema, Bologna, Il Mulino, 2009 (ebook)

6. Ball P., Shapes, Oxford, Oxford University Press, 2009

7. Baumgarten A.G., Aesthetica, Frankfurt/Oder, 1750

8. Beltrame R., La prospettiva rinascimentale, S.S.S., Roma, 1996

9. Benjamin W., L'opera d'arte nell'epoca della sua riproducibilità tecnica, Torino, Einaudi, 1966

10. Bensaude-Vincent B. \& Newman W.R., The Artificial and the Natural, Cambridge, Massachusetts, London, England, MIT Press, 2007

11. Bertin A., Poli M. \& Vitale A., Fondamenti di meccanica, Bologna, Progetto Leonardo, 1997

12. Bloomer K.C. \& Moore C.W., Corpo, memoria, architettura, Sansoni, 


\section{Firenze, 1981}

13. Bodei R., Le forme del bello, Bologna, Il Mulino, 1995

14. Bolzoni L., La stanza della memoria, Einaudi, Torino, 1995

15. Bozal V., Il gusto, Bologna, Il Mulino, 1996

16. Bussagli M., Il nudo nell'arte, Firenze, Giunti, 1998

17. Braidotti R., Metamorphoses. Towards a Matherialistic Theory of Becoming, Cambridge, Polity Press, 2002; trad. it., In metamorfosi. Verso una teoria materialistica del divenire, Milano, Feltrinelli, 2003

18. Burke E., A Philosophical Enquiry into the Origin of Our Ideas of the Sublime and Beautiful, 1757

19. Cappelletto C., Neuroestetica, Roma-Bari, Laterza, 2009

20. Caronia A., Il cyborg. Saggio sull'uomo artificiale, Roma-Napoli, Theoria, 1985

21. Carrol S.B., Infinite forme bellissime, Torino, Codice, 2006

22. Cascetta E., Metodi quantitativi per la pianificazione dei sistemi di trasporto, Padova, Cedam, 1990

23. Castellani E., Simmetria e natura. Dalle armonie delle figure alle invarianze delle leggi, Roma-Bari, Laterza, 2000

24. Castelli P., L'estetica del Rinascimento, Bologna, Il Mulino, 2005

25. Caygill H., A Kant Dictionary, Malden, USA, Oxford, UK, Victoria, AU,

Blackwell Publishing, 1995

26. Churchland P.M., La natura della mente e la struttura della scienza. Una prospettiva neurocomputazionale, Bologna, Il Mulino, 1992

27. Cole K.C., L'universo e la tazza da tè, Milano, Longanesi \& C., 1999

28. Costa M., Il sublime tecnologico. Piccolo trattato di estetica della tecnologia, Roma, Castelvecchi, 1998

29. D'Angelo P., L'estetica del Romanticismo, Il Mulino, Bologna, 1997

30. D'Angelo P., Estetismo, Bologna, Il Mulino, 2003

31. D'Angelo P., Estetica e paesaggio, Bologna, Il Mulino, 2009 (e-book)

32. Debord G., Commentari sulla società dello spettacolo, Milano, SugarCo, 1990

33. Deleuze G. \& Guattari F., Mille plateaux. Capitalisme et schizophrénie, Paris, Minuit, 1980; trad. it., Mille piani. Capitalismo e schizofrenia, Roma, Istituto Enciclopedia Italiana, 1987

34. Deleuze G., La piega. Leibniz e il Barocco, Torino, Einaudi, 2004 35. Dürer A., Della simmetria de i corpi humani (rist. anast. 1591), Aragno, 1999

36. Du Sautoy M., Il disordine perfetto. L'avventura di un matematico nei segreti della simmetria, BUR, 2010

37. Eco U., Kant e l'ornitorinco, Milano, Bompiani, 1997

38. Eco U., Storia della bruttezza, Milano, Bompiani, 2007

39. Eco U., Storia della bellezza, Milano, Bompiani, 2007

40. Fereidoun M. E., Are you a Transhuman?, London, Warnerbooks, 1989 41. Foucault M., Les mots et les choses. Une archéologie des sciences humaines, Paris, Gallimard, 1966; trad. it. Le parole e le cose. Un'archeologia delle scienze umane, Milano, Rizzoli, 1985

42. Francalanci E. L., Estetica degli oggetti, Bologna, Il Mulino, 2006

43. Franzini E., L'estetica del Settecento, Il Mulino, Bologna, 2002

44. Freud S., Al di là del principio del piacere, Varese, Bruno Mondadori, 1998

45. Fubini E., Estetica della musica, Bologna, Il Mulino, 1995

46. Fumagalli B. \& Brocchieri M., L'estetica medievale, Bologna, Il Mulino, 2002

47. Giacomoni P., Il laboratorio della natura, Paesaggio montano e sublime naturale in età moderna, Milano, Franco Angeli, 2001

48. Halberstam J. \& Livingston, I. (a cura), Posthuman Bodies, Bloomington, Indiana UP, 1995

49. Haraway D.J., Simians, Cyborgs and Women. The Reinvention of Nature, London, Routledge, 1991

50. Benamou M., Caramella C. (a cura), Performance in Postmodern Culture, Madison WI, Coda Press, 1977

51. Hayles K.N., How We Became Posthuman. Virtual Bodies in Cybernetics, Literature and Informatics, Chicago, University of Chicago Press, 1999

52. Hubel D., Occhio, cervello e visione, Bologna, Zanichelli, 1989

53. Kember S., Cyberfeminism and Artificial Life, London, Routledge, 2003,

54. Kobau P., Matteucci G. \& Velotti S. (a cura), Estetica e filosofia analitica, Bologna, Il Mulino, 2009 (e-book)

55. Lapucci C., Il numero e la struttura universale, Firenze, Polistampa, 2010

56. Leibniz G.W., Nuovi saggi sull'intelletto umano, 1765, Libro II, Cap. $1, \S 6$

57. Levi P., L'asimmetria e la vita. Articoli e saggi 1955-1987, Torino, Einaudi, 2002

58. Livio M., L'equazione impossibile. Come un genio della matematica ha scoperto il linguaggio della simmetria, Milano, Rizzoli, 2006

59. Locke J., An Essay Concerning Human Understanding, 1690, Libro II, Cap. $1, \S 5$

60. Lombardo G., L'estetica antica, Bologna, Il Mulino, 2002

61. Longo G.O., Il simbionte. Prove di umanità futura, Roma, Meltemi, 2003
62. Macrì T., Il corpo postorganico, Milano, Costa \& Nolan, 1996

63. Maffei L. \& Fiorentini A., Arte e cervello, Bologna, Zanichelli, 1995

64. Mantero A. M. \& Ferrari A., La Magia dei gruppi di simmetria. I gruppi dei magnifici rosoni, Firenze, Alinea, 2009

65. Marchesini R., Post-human. Verso nuovi modelli di esistenza, Torino, Bollati Boringhieri, 2002

66. Maturana H. R. \& Varela F. J., Autopoiesis and Cognition. The Realization of the Living. Boston Studies in the Philosophy of Science 1980;42, trad. it Autopoiesi e cognizione. La realizzazione del vivente, Venezia, Marsilio, 1985 67. Meinhardt H., The Algorithmic Beauty of Sea Shells, Berlin Heidelberg, Springer-Verlag, 2009

68. Merleau-Ponty M., Phénoménologie de la perception, Paris, Éditions Gallimard, 1945

69. Merleau-Ponty M., L'oeil et l'esprit, Parigi, 1964

70. Milani R., I volti della Grazia, Bologna, Il Mulino, 2009 (e-book)

71. Ministero delle Infrastrutture e dei Trasporti, Norme funzionali e geometriche per la costruzione delle strade, G.U. del 4/1/2002

72. Montani P., Estetica ed ermeneutica. Senso, contingenza, verità, RomaBari, Laterza, 2002

73. Moravec H., Mind Children. The Future of Robot and Human Intelligence, Cambridge, Harvard UP, 1990

74. Niro P., Ludwig Wittgenstein e la musica. Osservazioni filosofiche e riflessioni estetiche sul linguaggio musicale negli scritti di Ludwig Wittgenstein Napoli, ESI, 2008

75. Onfray M., L'arte di gioire. Per un materialismo edonista, Roma, Fazi, 2009 76. Perniola M., Il sex appeal dell'inorganico, Torino, Einaudi, 1994

77. Perniola M., Disgusti. Le nuove tendenze estetiche, Milano, Costa \& Nolan 1999

78. Perniola M., Del sentire, Torino, Einaudi, 2002

79. Pezzella M., Estetica del cinema, Bologna, Il Mulino, 1996

80. Plessner H., Studi di estesiologia. L'uomo, i sensi, il suono, Bologna, CLUEB, 2007

81. Radicati di Bronzolo L.A., Pensare la natura, Roma-Bari, Laterza, 1999

82. Rocca E., Estetica e architettura, Bologna, Il Mulino, 2009 (e-book)

83. Ronan M., Il mostro e la simmetria. Una delle più grandi scoperte della matematica, Milano, Raffaello Cortina, 2007

84. Roni R., La persistenza dell'istinto. Pulsioni vitali dell'esistenza, Pisa, ETS 2007

85. Rosenkranz K., Estetica del brutto, Milano, Olivares, 1994 (Aesthetik des Hässlichen", 1853)

86. Russo F., Modelli statistici per il calcolo di tempi e costi per il trasporto merci in Italia, Reggio Calabria, Quaderno della Facoltà di Ingegneria, n. 8, 1994

87. Saint Girons B., Il sublime, Bologna, Il Mulino, 2006

88. Sartwell C., I sei nomi della bellezza, Torino, Einaudi, 2006

89. Schattschneider D., Visioni della simmetria. I disegni periodici di M. C. Escher, Bologna, Zanichelli, 1992

90. Siani A.L., Kant e Platone. Dal mondo delle idee all'idea nel mondo, Pisa ETS, 2007

91. Shannon C. E., Weaver, W., 1949, The Mathematical Theory of Communication, Urbana, University of Illinois Press; trad. it., La teoria matematica delle comunicazioni, Milano, Etas, 1971

92. Snyder J. R., L'estetica del Barocco, Bologna, Il Mulino, 2005

93. Squier S. M., Babies in Bottles. Twentieth Century Visions of Reproductive Technology, New Brunswick, Rutgers UP, 1994

94. Stewart I., L'eleganza della verità. Storia della simmetria, Torino, Einaudi, 2008

95. Stone A. R., The War of Desire and Technology at the Close of the Mechanical Age, Cambridge Mass., MIT Press, 1995; trad. it., Desiderio e tecnologia. Il problema dell'identità nell'era di Internet, Milano, Feltrinelli, 1997

96. Tauber A. I., The Elusive Synthesis: Aesthetics and Science, DordrechtBoston-London, Kluwer Academic Publisher, 1997

97. Terrosi R., La filosofia del postumano, Milano, Costa \& Nolan, 1997

98. Tiezzi E., Beauty Science, Southampton, Boston, WIT Press, 2005

99. Tiezzi E., Verso una fisica evolutiva, Roma, Donzelli, 2006

100. Turing A. M., Computing Machinery and Intelligence. Mind 1950;54: 433-457

101. Tursi F., Estetica dei nuovi media, Milano, Costa \& Nolan, 2007

102. Varela F.J., Thompson E. \& Rosch E., The Embodied Mind. Cognitive Science and Human Experience, Cambridge, Mass., MIT Press, 1991; trad. it. La via di mezzo della conoscenza, Milano, Feltrinelli, 1992

103. Vercellone F., Bertinetto Alessandro \& Garelli Gianluca, Storia dell'estetica moderna e contemporanea, Bologna, Il Mulino, 2003

104. Vercellone F., Bertinetto A.o \& Garelli G., Lineamenti di storia dell'estetica, Bologna, Il Mulino, 2008

105. Vercellone F., Oltre la bellezza, Bologna, Il Mulino, 2008

106. Viganò V., A come Asimmetria, Roma, Gangemi, 2009

107. Vigarello G., Storia della bellezza, Roma, Donzelli, 2007

108. Wiener N., The Human Use of Human Beings, Boston, Houghton Mifflin Company, 1950; trad. it. Introduzione alla cibernetica. L'uso umano degli esseri umani, Torino, Bollati Boringhieri, 1966 